\title{
dDocent: a RADseq, variant-calling pipeline designed for population genomics of non-model organisms
}

Restriction-site associated DNA sequencing (RADseq) has become a powerful and useful approach for population genomics. Currently, no software exists that utilizes both paired-end reads from RADseq data to efficiently produce population-informative variant calls, especially for non-model organisms with large effective population sizes and high levels of genetic polymorphism. $d D o c e n t$ is an analysis pipeline with a user-friendly, command-line interface designed to process individually barcoded RADseq data (with double cut sites) into informative SNPs/Indels for population-level analyses. The pipeline, written in BASH, uses data reduction techniques and other stand-alone software packages to perform quality trimming and adapter removal, de novo assembly of RAD loci, read mapping, SNP and Indel calling, and baseline data filtering. Double-digest RAD data from population pairings of three different marine fishes were used to compare $d D o c e n t$ with Stacks, the first generally available, widely used pipeline for analysis of RADseq data. $d$ Docent consistently identified more SNPs shared across greater numbers of individuals and with higher levels of coverage. This is due to the fact that $d D o c e n t$ quality trims instead of filtering and incorporates both forward and reverse reads in assembly, mapping, and SNP calling, thus enabling use of reads with Indel polymorphisms. The pipeline and a comprehensive user guide can be found at ( http://dDocent.wordpress.com ). 
1 dDocent: a RADseq, variant-calling pipeline designed for population genomics of non-model organisms

\author{
Jonathan B. Puritz†, Christopher M. HollenbeCK, AND JOHN R. Gold
}

4 Marine Genomics Laboratory, Harte Research Institute, Texas A\&M University-Corpus Christi,

56300 Ocean Drive, Corpus Christi, Texas 78412-5869

$6 \uparrow$ Author to whom correspondence should be addressed.

7 Email: jonathan.puritz@tamucc.edu Phone: 361-825-3343 Fax: 361-825-2050 
Next-generation sequencing (NGS) has transformed the field of genetics into genomics by

10 providing DNA sequence data at an ever increasing rate and reduced cost (Mardis, 2008). The

11 nascent field of population genomics relies on NGS coupled with laboratory methods to

12 reproducibly reduce genome complexity to a few thousand loci. The most common approach,

13 restriction-site associated DNA sequencing (RADseq), uses restriction endonucleases to

14 randomly sample the genome at locations adjacent to restriction-enzyme recognition sites that,

15 when coupled with Illumina sequencing, produces high coverage of homologous SNP (Single

16 Nucleotide Polymorphism) loci. As such, RADseq provides a powerful method for population

17 level genomic studies (Ellegren, 2014;Narum et al., 2013;Rowe et al., 2011).

18 The original RADseq approach (Baird et al., 2008), and initial population genomic studies

19 employing it (Hohenlohe et al., 2010), focused on SNP discovery and genotyping on the first

20 (forward) read only. This is because the original RADseq method (Baird et al., 2008) utilized

21 random shearing to produce RAD loci; paired-end reads were not of uniform length or coverage,

22 making it problematic to find SNPs at high and uniform levels of coverage across a large

23 proportion of individuals. As a result, the most comprehensive and widely used software package

24 for analysis of RADseq data, Stacks (Catchen et al., 2013, 2011), provides SNP genotypes based

25 only on first-read data. In contrast, RADseq approaches such as ddRAD (Peterson et al., 2012),

26 2bRAD (Wang et al., 2012), and ezRAD (Toonen et al., 2013) rely on restriction enzymes to

27 define both ends of a RAD locus, largely producing RAD loci of fixed length (flRAD). Paired-

28 end Illumina sequencing of flRAD fragments provides an opportunity to significantly expand the

29 number of SNPs that can be genotyped from a single RADseq library.

30 Here, the variant-calling pipeline $d$ Docent is introduced as a tool for generating population

31 genomic data; a brief methodological outline of the analysis pipeline also is presented. dDocent

32 is a wrapper script designed to take raw flRAD data and produce population informative SNP 
33 calls (SNPs that are shared across the majority of individuals and populations), taking full

34 advantage of both paired-end reads. dDocent is configured for organisms with high levels of

35 nucleotide and INDEL polymorphisms, such as are found in many marine organisms (Guo et al.,

36 2012;Keever et al., 2009; Sodergren et al., 2006;Waples, 1998;Ward et al., 1994); however, the

37 pipeline also can be adjusted for low polymorphism species. As input, dDocent takes paired

38 FASTQ files for individuals and outputs raw SNP and INDEL calls as well as filtered SNP calls in

39 VCF format. The pipeline and a comprehensive online manual can be found at

40 (http://dDocent.wordpress.com). Finally, results of pipeline analyses, using both $d$ Docent and

41 Stacks, of populations of three species of marine fishes are provided to demonstrate the utility of

42 dDocent compared to Stacks, the first and most comprehensive, existing software package for

43 RAD population genomics.

\section{METHODS}

Implementation and basic usage

46 The $d$ Docent pipeline is written in BASH and will run using most Unix-like operating

47 systems. dDocent is largely dependent on other bioinformatics software packages, taking

48 advantage of programs designed specifically for each task of the analysis and ensuring that each

49 modular component can be updated separately. Proper implementation depends on the correct

50 installation of each third-party packages/tools. A full list of dependencies can be found in the

51 user manual at (http://ddocent.wordpress.com/ddocent-pipeline-user-guide/) and a sample script

52 to automatically download and install the packages in a Linux environment can be found at the

53 dDocent repository (https://github.com/jpuritz/dDocent).

$54 d$ Docent is run by simply switching to a directory containing input data and starting the

55 program. There is no configuration file, and dDocent will proceed through a short series of

56 command-line prompts, allowing the user to establish analysis parameters. After all required

57 variables are configured, including an e-mail address for a completion notification, dDocent 
58 provides instructions on how to move the program to the background and run, undisturbed, until

59 completion. The pipeline is designed to take advantage of multiple processing-core machines

60 and, whenever possible, processes are invoked with multiple threads or occurrences. For most

61 Linux distributions, the number of processing cores should be automatically detected. If $d$ Docent

62 cannot determine the number of processors, it will ask the user to input the value.

63 There are two distinct modules of dDocent: dDocent.FB and dDocent.GATK. dDocent.FB

64 uses minimal, BAM-file preparation steps before calling SNPs and INDELs, simultaneously using

65 FreeBayes (Garrison \& Marth, 2012). dDocent.GATK uses GATK (McKenna et al., 2010) for

66 INDEL realignment, SNP and INDEL genotyping (using HaplotypeCaller), and variant quality-

67 score recalibration, largely following GATK Best Practices recommendations (Auwera \&

68 Carneiro, 2013;DePristo et al., 2011). The modules represent two different strategies for

69 SNP/INDEL calling that are completely independent of one another. Currently, dDocent.FB is

70 easier to implement, substantially faster to execute, and depends on software that is commercially

71 unrestricted; consequently, the remainder of this paper focuses on dDocent.FB. Additional

72 information on dDocent.GATK may be found in the user guide.

73 Data input requirements

74 dDocent requires demultiplexed forward and paired-end FASTQ files for every individual in

75 the analysis (flRAD data only). A simple naming convention (a single-word locality code/name

76 and a single-word sample identifier separated by an underscore) must be followed for every

77 sample; examples are LOCA_IND01.F.fq and LOCA_IND01.R.fq. A sample script for using a

78 text file containing barcodes and sample names and process_radtags from Stacks (Catchen et al.,

79 2013) to properly demultiplex samples and put them in the proper $d$ Docent naming convention,

80 can be found at the $d$ Docent repository (https://github.com/jpuritz/dDocent).

81 Quality trimming 
82 After $d$ Docent checks that it is recognizing the proper number of samples in the current

83 directory, it asks the user if $\mathrm{s} / \mathrm{he}$ wishes to proceed with quality trimming of sequence data. If

84 directed, dDocent can use the program Trim Galore!

85 (http://www.bioinformatics.babraham.ac.uk/projects/trim_galore/) to simultaneously remove

86 Illumina adapter sequences and trim ends of reads of low quality. By default, Trim Galore! looks

87 for double-digest RAD adapters (Peterson et al., 2012) and trims bases with quality scores less

88 than PHRED 10 (corresponding to a 10\% chance of error in the base call). The read mapping and

89 variant calling steps of $d$ Docent account for base quality, so minimal trimming of the data is

90 needed. Typically, quality trimming only needs to be performed once, so the option exists to skip

91 this step in subsequent $d$ Docent analyses.

92 De novo assembly

93 Without reference material, population genomic analyses from RADseq depend on de novo

94 assembly of a set of reference contigs. Intrinsically, not all RAD loci appear in all individuals

95 due to stochastic processes inherent in library preparation and sequencing and to polymorphism

96 in restriction-enzyme restriction sites (Catchen et al., 2011). Moreover, populations can contain

97 large levels of within-locus polymorphism, making generation of a reference sequence

98 computationally difficult. dDocent minimizes the amount of data used for assembly by taking

99 advantage of the fact that flRAD loci present in multiple individuals should have higher levels of

100 exactly matching reads (forward and reverse) than loci that are only present in a few individuals.

101 Caution is advised for unique reads with low levels of coverage throughout the data set as they

102 likely represent sequencing errors or polymorphisms that are shared only by a few individuals.

103 In the first step of the assembly process, untrimmed, paired-end reads are reverse

104 complemented and concatenated to forward reads. Unique paired reads are identified and their

105 occurrences are counted in the entire data set. These data are tabulated into the number of unique

106 reads per levels of $1 \mathrm{X}$ to $50 \mathrm{X}$ coverage; a graph is then generated and printed to the terminal. 
107 The distribution usually follows an asymptotic relationship (Figure 1), with a large proportion of

108 reads only having one or two occurrences, meaning they likely will not be informative on a

109 population scale. Highly polymorphic RAD loci still should have at least one allele present at the

110 level of expected sequence coverage, so this can be used as a guide for informative data. The

111 user chooses a cut-off level of coverage for reads to be used for assembly - note that all reads are

112 still used for subsequent steps of the pipeline.

113 After a cut-off level is chosen, remaining concatenated reads are divided back into forward-

114 and reverse-read files and then input directly into the RADseq assembly program Rainbow

115 (Chong et al., 2012). The default parameters of Rainbow are used except that the maximum

116 number of mismatches used in initial clustering is changed from four to six to help account for

117 highly polymorphic species with large effective population sizes. In short, Rainbow clusters

118 forward reads based on similarity; clusters are then recursively divided, based on reverse reads,

119 into groups representing single alleles. Reads in merged clusters are then assembled using a

120 greedy algorithm (Pop \& Salzberg, 2008). dDocent then selects the longest contig for each

121 cluster as the representative reference sequence for that RAD locus. If the forward read does not

122 overlap with the reverse read (almost always the case with flRAD), the forward read is

123 concatenated to the reverse read with ten ' $N$ ' characters as padding to represent the unknown

124 insert. If forward and reverse reads do overlap, then a full contig is created without $\mathrm{N}$ padding.

125 Finally, reference sequences are clustered based on overall sequence similarity (chosen by user,

$12690 \%$ by default), using the program CD-HIT (Fu et al., 2012;Li \& Godzik, 2006). This final

127 cluster step reduces the data set further, based on overall sequence identity after assembly.

128 Alternatively, de novo assembly can be skipped and the user can provide a FASTA file with

129 reference sequences.

130 Read mapping 
132 quality-trimmed reads to the reference contigs. Users can deploy the default values of BWA or

133 set an alternative value for each mapping parameter (match score, mismatch score, and gap-

134 opening penalty). The default settings are meant for mapping reads to the human genome, so

135 users are encouraged to experiment with mapping parameters. BWA output is ported to

136 SAMtools (Li et al., 2009), saving disk space, and alignments are saved to the disk as binary

137 alignment/Map (BAM). BAM files are then sorted and indexed.

138 SNP and INDEL discovery and genotyping

139 dDocent uses a two-step process to optimize the computationally intensive task of

140 SNP/INDEL calling. First, quality-trimmed forward and reverse reads are reduced to unique

141 reads. This data set is then mapped to all reference sequences, using the previously entered

142 mapping settings (see Read Mapping above). From this alignment, a set of intervals is created

143 using BEDtools (Quinlan \& Hall, 2010). The interval set saves computational time by directing

144 the SNP-/INDEL-calling software to examine only reference sequences along contigs that have

145 high quality mappings. Second, the interval list is then split into multiple files, one for each

146 processing core, allowing SNP/INDEL calling to be optimized with a scatter-gather technique.

147 The program FreeBayes (Garrison \& Marth, 2012) is then executed multiple times

148 simultaneously (one execution per processor and genomic interval). FreeBayes is a Bayesian-

149 based, variant-detection software that uses assembled haplotype sequences to simultaneously call

150 SNPs, INDELS, multi-nucleotide polymorphisms (MNPs), and complex events (e.g., composite

151 insertion and substitution events) from alignment files; FreeBayes has the added benefit for

152 population genomics of using reads across multiple individuals to improve genotyping (Garrison

$153 \&$ Marth, 2012). FreeBayes is run with minimal changes to the default parameters; minimum

154 mapping quality score and base quality score are set to PHRED 10. After all executions of 
155 FreeBayes are completed, raw SNP/INDEL calls are concatenated into a single variant call file

156 (VCF), using VCFtools (Danecek et al., 2011).

\section{Variant Filtering}

158 Final SNP data-set requirements are likely to be highly dependent on specific goals and aims

159 of individual projects. To that end, dDocent uses VCFtools (Danecek et al., 2011) to provide only

160 basic level filtering, mostly for run diagnostic purposes. $\mathrm{d}$ Docent produces a final VCF file that

161 contains all SNPs, INDELS, MNPs, and complex events that are called in $90 \%$ of all individuals,

162 with a minimum quality score of 30 . Users are encouraged to use VCFtools and vcflib (part of

163 the FreeBayes package; https:/github.com/ekg/vcflib) to fully explore and filter data

164 appropriately.

165 Comparison between dDocent and Stacks

166 Two sample localities, each comprising 20 individuals, were chosen randomly from

167 unpublished RADseq data sets of three different, marine fish species: red snapper (Lutjanus

168 campechanus), red drum (Sciaenops ocellatus), and silk snapper (Lutjanus vivanus). These three

169 species are part of ongoing RADseq projects in our laboratory, and preliminary analyses

170 indicated high levels of nucleotide polymorphisms across all populations. Double-digest RAD

171 libraries were prepared, generally following Peterson et al. (2012). Individual DNA extractions

172 were digested with EcoRI and MspI. A barcoded adapter was ligated to the EcoRI site of each

173 fragment and a generic adapter was ligated to the MspI site. Samples were then equimollarly

174 pooled and size-selected between 350 and 400 bp, using a Qiagen Gel Extraction Kit. Final

175 library enhancement was completed using 12 cycles of PCR, simultaneously enhancing properly

176 ligated fragments and adding an Illumina Index for additional barcoding. Libraries were

177 sequenced on three separate lanes of an Illumina HiSeq 2000 at the University of Texas Genomic

178 Sequencing and Analysis Facility. Raw sequence data were archived at NCBI's Short Read

179 Archive (SRA) under Accession SRP041032. 

different levels of final reference contig clustering (90\%, 96\%, and 99\% similarity) in an attempt to alter the most comparable analysis variable in $d$ Docent to match the maximum distance

183 between stacks parameter and the maximum distance between stacks from different individuals 184 parameter of Stacks. The coverage cut-off for assembly was 12 for red snapper, 13 for red drum, 185 and nine for silk snapper. All dDocent runs used mapping variables of one, three, and five for match-score value, mismatch score, and gap-opening penalty, respectively. For comparisons, complex variants were decomposed into canonical SNP and INDEL representation from the raw VCF files, using vcfallelicprimitives from vcflib (https://github.com/ekg/vcflib). process_radtags, removing reads with ' $\mathrm{N}$ ' calls and low-quality base scores. Because $d$ Docent inherently uses both reads for SNP/INDEL genotyping, forward reads and reverse reads were processed separately with denovo_map.pl, using three different sets of parameters. The first set had a minimum depth of coverage of two to create a stack, a maximum distance of two between stacks, and a maximum distance of four between stacks from different individuals, with both the deleveraging algorithm and removal algorithms enabled. The second set had a minimum depth of coverage of three to create a stack, a maximum distance of four between stacks, and a maximum distance of eight between stacks from different individuals, with both the deleveraging algorithm and removal algorithms enabled. The third set had a minimum depth of coverage of three to create a stack, a maximum distance of four between stacks, and a maximum distance of 10

200 between stacks from different individuals, with both the deleveraging algorithm and removal 201 algorithms enabled. SNP calls were output in VCF format.

202 For both dDocent and Stacks runs, VCFtools was used to filter out all INDELs and SNPs that 203 had a minor allele count of less than five. SNP calls were then evaluated at different individual204 coverage levels: the total number of SNPs; the number of SNPs called in $75 \%, 90 \%$, and $99 \%$ of 
205 individuals at $3 \mathrm{X}$ coverage; the number of SNPs called in $75 \%$ and $90 \%$ of individuals at $5 \mathrm{X}$

206 coverage; the number of SNPs called in $75 \%$ and $90 \%$ of individuals at $10 \mathrm{X}$ coverage; and the

207 number of SNPS called in $75 \%$ and $90 \%$ of individuals at $20 \mathrm{X}$ coverage. Overall coverage levels

208 for red snapper were lower and likely impacted by a few low-quality individuals; consequently,

209 the number of $5 \mathrm{X}$ and 10X SNPs shared among 90\% of individuals (after removing the bottom

$21010 \%$ of individuals in terms of coverage) were compared instead of SNP loci shared at 20X

211 coverage. Results from two runs of Stacks (one using forward and one using reverse reads) were

212 combined for comparison with $d$ Docent, which inherently calls SNPs on both reads. All analyses

213 and computations were performed on a 32-core Linux workstation with 128 GB of RAM.

\section{RESULTS AND DISCUSSION}

215 Results of SNP calling, including run times (in minutes) for each analysis (not including

216 quality trimming), are presented in Table 1. Data from high coverage SNP calls, averaged over

217 all runs for each pipeline, are presented in Figure 1. While Stacks called a larger number of low

218 coverage SNPs, limiting results to higher individual coverage and to higher individual call rates

219 revealed that $d$ Docent consistently called more high-quality SNPs. Run times were equivalent

220 for both pipelines.

221 At almost all levels of coverage in three different data sets, $d$ Docent called more SNPs across

222 more individuals than Stacks. Two key differences between dDocent and Stacks likely contribute

223 these discrepancies: (i) quality trimming instead of quality filtering, and (ii) simultaneous use of

224 forward and reverse reads by dDocent in assembly, mapping, and genotyping, instead of

225 clustering as employed by Stacks. As with any data analysis, quality of data output is directly

226 linked to the quality of data input. Both dDocent and Stacks use procedures to ensure that only

227 high-quality sequence data are retained; however, Stacks removes an entire read when a sliding

228 window of bases drops below a preset quality score (PHRED 10, by default), while $d$ Docent via

229 Trim Galore! trims off low-quality bases, preserving high-quality bases of each read. Filtering 
instead of trimming results in fewer reads entering the Stacks analysis (between 65\%-95\% of the

231 data compared to $d$ Docent; data not shown), generating lower levels of coverage and fewer SNP

232 calls than $d$ Docent.

$233 d$ Docent offers two advantages over Stacks: (i) it is specifically designed for paired-end data

234 and utilizes both forward and reverse reads for de novo RAD loci assembly, read mapping,

235 variant discovery, and genotyping; and (ii) it aligns reads to reference sequence instead of

236 clustering by identity. Using both reads to cluster and assemble RAD loci helps to ensure that

237 portions of the genome with complex mutational events, including INDELs or small repetitive

238 regions, are properly assembled and clustered as homologous loci. Additionally, using $B W A$ to

239 map reads to reference loci enables $d$ Docent to properly align reads with INDEL polymorphisms,

240 increasing coverage and subsequent variant discovery and genotyping. Clustering methods

241 employed by Stacks, whether clustering alleles within an individual or clustering loci between

242 individuals, effectively remove reads, alleles, and loci with INDEL polymorphisms because the

243 associated frame shift effectively inflates the observed number of base-pair differences. For

244 organisms with large effective population sizes and high levels of genetic diversity, such as many

245 marine organisms (Waples, 1998; Ward et al., 1994), removing reads and loci with INDEL

246 polymorphisms will result in a loss of shared loci and coverage.

$247 d$ Docent is specifically designed to efficiently generate SNP and INDEL polymorphisms that

248 are shared across multiple individuals. To that end, the output reference contigs and variant calls

249 represent a subset of the total, genomic information content of the raw input data; RAD loci and

250 variants present in single individuals are largely ignored. Other analysis software, such as the

251 scripts published by Peterson et al. (2012), represent a more comprehensive alternative for

252 generating for a full de novo assembly of RAD loci and would increase the chance of discovering

253 individual level polymorphisms. For population genomics, loci that are not shared by at least

$25450 \%$ of all individuals and/or have minor allele frequencies of less than $5 \%$ are often filtered out. 
255 dDocent saves computational time by ignoring these loci from the outset of assembly; however,

256 users can pass in a more comprehensive reference (including an entire genome) in order to

257 include all possible variant calls from the data.

\section{CONCLUSION}

$259 d$ Docent is an open-source, freely available population genomics pipeline configured for

260 species with high levels of nucleotide and INDEL polymorphisms, such as many marine

261 organisms. The $d$ Docent pipeline reports more SNPs shared across greater numbers of

262 individuals and with higher levels of coverage than current alternatives. The pipeline and a

263 comprehensive online manual can be found at (http://dDocent.wordpress.com) and

264 (https://github.com/jpuritz/dDocent).

\section{ACKNOWLEDGEMENTS}

266 We thank T. Krabbenhoft for assistance in beta testing, and C. Bird and D. Portnoy for useful 267 discussions and comments on the manuscript. We also would like to thank the three reviewers

268 for their substantial help with troubleshooting the user guide and installation process on multiple 269 computing platforms. 
271

272

273

274

275

276

277

278

279

280

281

282

283

284

285

286

287

288

289

290

291

292

293

294

295

296

297

298

299

300

301

302

303

304

305

306

307

308

309

310

311

312

313

314

Auwera G, Carneiro M. 2013. From FastQ Data to High-Confidence Variant Calls: The Genome Analysis Toolkit Best Practices Pipeline. Current Protocols in Bioinformatics: 1-33.

Baird NA, Etter PD, Atwood TS, Currey MC, Shiver AL, Lewis ZA, Selker EU, Cresko WA, Johnson EA. 2008. Rapid SNP discovery and genetic mapping using sequenced RAD markers. PloS ONE 3: e3376.

Catchen JM, Amores A, Hohenlohe P, Cresko W, Postlethwait JH. 2011. Stacks: building and genotyping Loci de novo from short-read sequences. G3 (Bethesda, Md.) 1: 171-182.

Catchen J, Hohenlohe PA, Bassham S, Amores A, Cresko WA. 2013. Stacks: an analysis tool set for population genomics. Molecular ecology 22: 3124-3140.

Chong Z, Ruan J, Wu C. 2012. Rainbow : an integrated tool for efficient clustering and assembling RAD-seq reads. : $1-6$.

Danecek P, Auton A, Abecasis G, Albers CA, Banks E, DePristo MA, Handsaker RE, Lunter G, Marth GT, Sherry ST, McVean G, Durbin R. 2011. The variant call format and VCFtools. Bioinformatics (Oxford, England) 27: 2156-2158.

DePristo MA, Banks E, Poplin R, Garimella K V, Maguire JR, Hartl C, Philippakis AA, Angel G del, Rivas MA, Hanna M, McKenna A, Fennell TJ, Kernytsky AM, Sivachenko AY, Cibulskis K, Gabriel SB, Altshuler D, Daly MJ. 2011. A framework for variation discovery and genotyping using next-generation DNA sequencing data. Nature genetics 43: 491-498.

Ellegren H. 2014. Genome sequencing and population genomics in non-model organisms. Trends in ecology \& evolution 29: 51-63.

Fu L, Niu B, Zhu Z, Wu S, Li W. 2012. CD-HIT: accelerated for clustering the next-generation sequencing data. Bioinformatics (Oxford, England) 28: 3150-3152.

Garrison E, Marth G. 2012. Haplotype-based variant detection from short-read sequencing. : 1-9.

Guo B, Zou M, Wagner A. 2012. Pervasive indels and their evolutionary dynamics after the fishspecific genome duplication. Molecular biology and evolution 29: 3005-3022.

Hohenlohe PA, Bassham S, Etter PD, Stiffler N, Johnson EA, Cresko WA. 2010. Population genomics of parallel adaptation in threespine stickleback using sequenced RAD tags. PLoS genetics 6: e1000862.

Keever CC, Sunday J, Puritz JB, Addison JA, Toonen RJ, Grosberg RK, Hart MW. 2009. Discordant distribution of populations and genetic variation in a sea star with high dispersal potential. Evolution; international journal of organic evolution 63: 3214-3227.

Li H, Durbin R. 2009. Fast and accurate short read alignment with Burrows-Wheeler transform. Bioinformatics (Oxford, England) 25: 1754-1760.

Li H, Durbin R. 2010. Fast and accurate long-read alignment with Burrows-Wheeler transform. Bioinformatics (Oxford, England) 26: 589-595.

Li W, Godzik A. 2006. Cd-hit: a fast program for clustering and comparing large sets of protein or nucleotide sequences. Bioinformatics (Oxford, England) 22: 1658-1659.

Li H, Handsaker B, Wysoker A, Fennell T, Ruan J, Homer N, Marth G, Abecasis G, Durbin R. 2009. The Sequence Alignment/Map format and SAMtools. Bioinformatics (Oxford, England) 25: 2078-2079.

Li H. 2013. Aligning sequence reads, clone sequences and assembly contigs with BWA-MEM. $00: 1-3$.

Mardis ER. 2008. Next-generation DNA sequencing methods. Annual review of genomics and human genetics 9: 387-402. 
315

316

317

318

319

320

321

322

323

324

325

326

327

328

329

330

331

332

333

334

335

336

337

338

339

340

341

342

McKenna A, Hanna M, Banks E, Sivachenko A, Cibulskis K, Kernytsky A, Garimella K, Altshuler D, Gabriel S, Daly M, DePristo MA. 2010. The Genome Analysis Toolkit: a MapReduce framework for analyzing next-generation DNA sequencing data. Genome research 20: 1297-1303.

Narum SR, Buerkle CA, Davey JW, Miller MR, Hohenlohe PA. 2013. Genotyping-by-sequencing in ecological and conservation genomics. Molecular ecology 22: 2841-2847.

Peterson BK, Weber JN, Kay EH, Fisher HS, Hoekstra HE. 2012. Double digest RADseq: an inexpensive method for de novo SNP discovery and genotyping in model and non-model species. PloS one 7: e37135.

Pop M, Salzberg S. 2008. Bioinformatics challenges of new sequencing technology. Trends in Genetics 24: 142-149.

Quinlan AR, Hall IM. 2010. BEDTools: a flexible suite of utilities for comparing genomic features. Bioinformatics (Oxford, England) 26: 841-842.

Rowe HC, Renaut S, Guggisberg A. 2011. RAD in the realm of next-generation sequencing technologies. Molecular ecology 20: 3499-3502.

Sodergren E, Weinstock GM, Davidson EH, Cameron RA, Gibbs RA, Angerer RC, Angerer LM, Arnone MI, Burgess DR, Burke RD, Coffman JA, Dean M, Elphick MR, Ettensohn CA, Foltz KR, Hamdoun A, Hynes RO, Klein WH, Marzluff W, et al. 2006. The genome of the sea urchin Strongylocentrotus purpuratus. Science (New York, N.Y.) 314: 941-952.

Toonen RJ, Puritz JB, Forsman ZH, Whitney JL, Fernandez-Silva I, Andrews KR, Bird CE. 2013. ezRAD: a simplified method for genomic genotyping in non-model organisms. PeerJ 1: e203.

Wang S, Meyer E, McKay JK, Matz M V. 2012. 2b-RAD: a simple and flexible method for genome-wide genotyping. Nature methods 9: 808-810.

Waples RS. 1998. Separating the wheat from the chaff: patterns of genetic differentiation in high gene flow species. Journal of Heredity 89: 438-450.

Ward RD, Woodwark M, Skibinski DOF. 1994. A comparison of genetic diversity levels in marine, freshwater, and anadromous fishes. Journal of Fish Biology 44: 213-232. 


\section{Figure 1}

Levels of coverage for each unique read in the red snapper data set.

The horizontal axis represents the minimal level of coverage, while the vertical axis represents the number of unique paired reads in thousands.

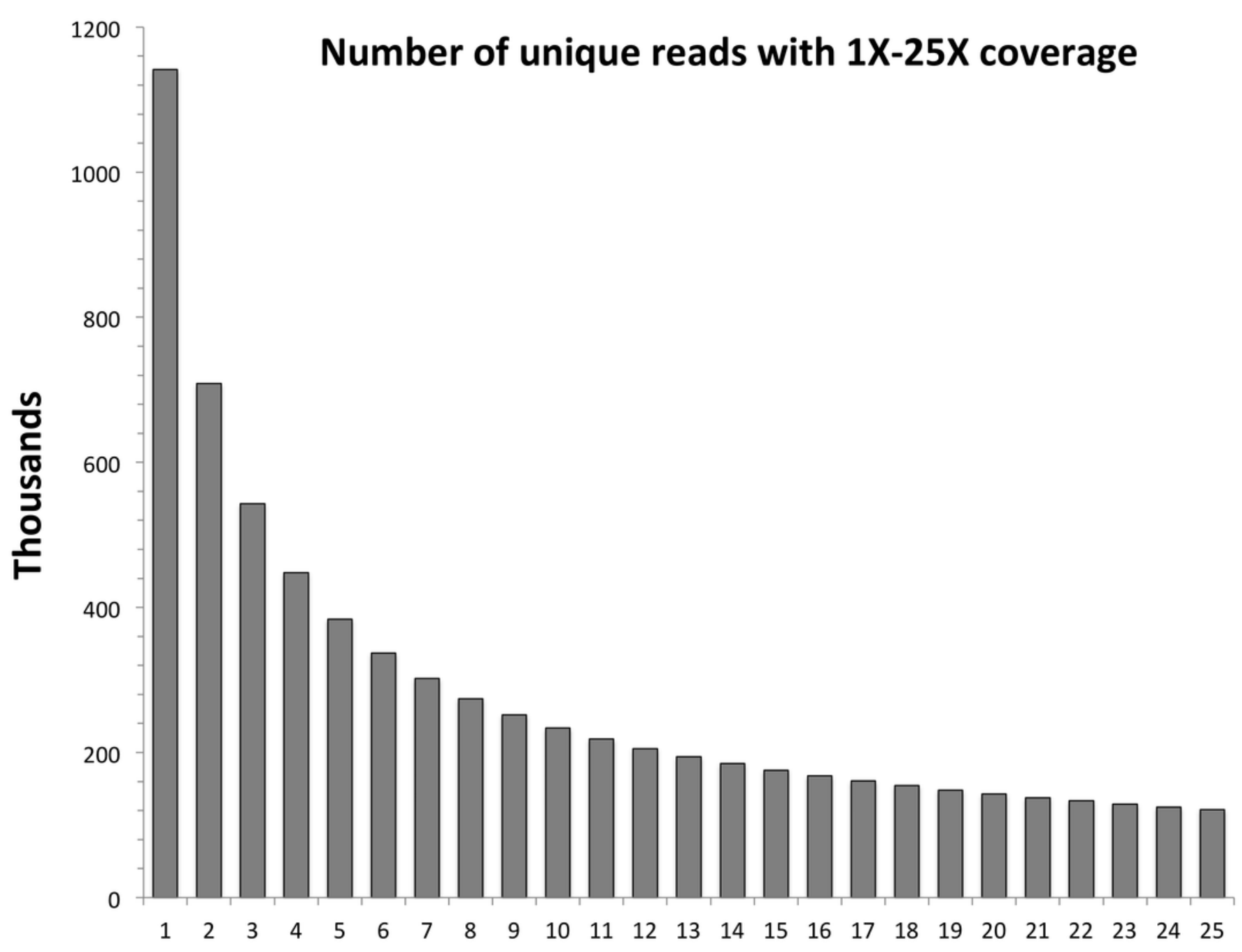




\section{Table $\mathbf{1}_{\text {(on next page) }}$}

Results from individual runs of $d D o c e n t$ and Stacks.

dDocent runs varied in the level of similarity used to cluster reference sequences: A $(90 \%)$, B (96\%), and C (99\%). For Stacks, forward reads and reverse reads were separately processed with denovo_map.pl (Stacks version 1.08), using three different sets of parameters: A, minimum depth of coverage of two to create a stack, a maximum distance of two between stacks, and a maximum distance of four between stacks from different individuals; $B$, minimum depth of coverage of three to create a stack, a maximum distance of four between stacks, and a maximum distance of eight between stacks from different individuals; and $\mathrm{C}$, minimum depth of coverage of three to create a stack, a maximum distance of four between stacks, and a maximum distance of 10 between stacks from different individuals. For $d D o c e n t$, complex variants were decomposed into canonical SNP and Indel calls and Indel calls were filtered out. SNP calls were evaluated at different individual coverage levels: (i) total number of SNPs; (ii) number of SNPS called in $75 \%, 90 \%$, and $99 \%$ at $3 X$ coverage; (iii) number of SNPS called in $75 \%$ and $90 \%$ of individuals at $5 X$ coverage; (iv) number of SNPS called in $75 \%$ and $90 \%$ of individuals at $10 \mathrm{X}$ coverage; and, (v) number of SNPS called in $75 \%$ and $90 \%$ of individuals at $20 \mathrm{X}$ coverage. Run times are in minutes. Results from forward and reverse reads of Stacks were combined for comparison with $d D o c e n t$, which inherently calls SNPs on both reads. 
Table 1. Results from individual runs of dDocent and Stacks. dDocent runs varied in the level of similarity used to cluster reference sequences: A (90\%), B (96\%), and C (99\%). For Stacks, forward reads and reverse reads were separately processed with denovo_map.pl (Stacks version 1.08), using three different sets of parameters: A, minimum depth of coverage of two to create a stack, a maximum distance of two between stacks, and a maximum distance of four between stacks from different individuals; B, minimum depth of coverage of three to create a stack, a maximum distance of four between stacks, and a maximum distance of eight between stacks from different individuals; and $\mathrm{C}$, minimum depth of coverage of three to create a stack, a maximum distance of four between stacks, and a maximum distance of 10 between stacks from different individuals. For $d$ Docent, complex variants were decomposed into canonical SNP and INDEL calls and INDEL calls were filtered out. SNP calls were evaluated at different individual coverage levels: (i) total number of SNPs; (ii) number of SNPS called in $75 \%, 90 \%$, and $99 \%$ at $3 X$ coverage; (iii) number of SNPS called in $75 \%$ and $90 \%$ of individuals at $5 \mathrm{X}$ coverage; (iv) number of SNPS called in $75 \%$ and $90 \%$ of individuals at $10 \mathrm{X}$ coverage; and, (v) number of SNPS called in $75 \%$ and $90 \%$ of individuals at $20 \mathrm{X}$ coverage. Run times are in minutes. Results from forward and reverse reads of Stacks were combined for comparison with dDocent, which inherently calls SNPs on both reads.

dDocent A dDocent B dDocent C Stacks A Stacks B Stacks C

\begin{tabular}{l|ccc|ccc} 
& \multicolumn{7}{c}{ Red snapper } \\
Total 3X SNPS & 53,298 & 53,316 & 53,361 & 28,817 & 33,479 & 53,298 \\
$75 \%$ 3X SNPs & 21,195 & 20,990 & 20,724 & 4,150 & 5,735 & 21,195 \\
$90 \%$ 3X SNPs & 9,102 & 8,850 & 8,639 & 675 & 987 & 9,102 \\
$99 \%$ 3X SNPs & 78 & 47 & 15 & - & - & 78
\end{tabular}




\begin{tabular}{|c|c|c|c|c|c|c|}
\hline $75 \%$ 5X SNPs & 14,881 & 14,594 & 14,339 & 2,632 & 4,351 & 14,881 \\
\hline $90 \% 5 X$ SNPs & 5,021 & 4,925 & 4,785 & 179 & 579 & 5,021 \\
\hline $75 \%$ 10X SNPs & 7,556 & 7,318 & 7,154 & 783 & 1,618 & 7,556 \\
\hline $90 \%$ 10X SNPS & 1,414 & 1,340 & 1,286 & 7 & 48 & 1,414 \\
\hline $90 \%$ IND $90 \% 5 \mathrm{X}$ & 10,267 & 10,026 & 9,798 & 806 & 1,807 & 10,267 \\
\hline $90 \%$ IND 90\% 10x & 4,242 & 4,093 & 3,974 & 129 & 441 & 4,242 \\
\hline Run time & 41 & 41 & 42 & 70 & 47 & 41 \\
\hline & \multicolumn{6}{|c|}{ Red drum } \\
\hline Total 3X SNPS & 46,378 & 46,688 & 46,832 & 45,792 & 50,821 & 46,378 \\
\hline $75 \% 3 \mathrm{X}$ SNPs & 36,745 & 36,905 & 36,900 & 24,134 & 28,991 & 36,745 \\
\hline $90 \% 3 \mathrm{X}$ SNPs & 32,356 & 32,424 & 32,330 & 13,439 & 17,946 & 32,356 \\
\hline $99 \% 3 X$ SNPs & 11,906 & 11,910 & 11,774 & 828 & 1,264 & 11,906 \\
\hline $75 \%$ 5X SNPs & 34,279 & 34,393 & 34,336 & 21,021 & 26,526 & 34,279 \\
\hline $90 \% 5 \mathrm{X}$ SNPs & 28,532 & 28,566 & 28,431 & 10,494 & 15,282 & 28,532 \\
\hline 75\% 10X SNPs & 27,523 & 27,605 & 27,488 & 12,928 & 17,018 & 27,523 \\
\hline $90 \% 10 X$ SNPS & 19,434 & 19,442 & 19,283 & 4,159 & 6,734 & 19,434 \\
\hline $75 \%$ 20X SNPs & 15,080 & 15,111 & 14,981 & 2,276 & 3,538 & 15,080 \\
\hline $90 \% 20 \mathrm{X}$ SNPs & 7,365 & 7,409 & 7,304 & 243 & 1,974 & 7,365 \\
\hline \multirow[t]{2}{*}{ Run time } & 43 & 45 & 45 & 58 & 55 & 43 \\
\hline & \multicolumn{6}{|c|}{ Silk snapper } \\
\hline Total 3X SNPS & 68,668 & 68,825 & 68,861 & 48,742 & 55,505 & 68,668 \\
\hline $75 \% 3 X$ SNPs & 30,771 & 30,391 & 30,051 & 7,596 & 9,705 & 30,771 \\
\hline $90 \% 3 \mathrm{X}$ SNPs & 14,952 & 14,673 & 14,415 & 2,007 & 3,439 & 14,952 \\
\hline $99 \% 3 X$ SNPs & 4,294 & 4,060 & 3,952 & 132 & 527 & 4,294 \\
\hline 75\% 5X SNPs & 20,534 & 20,188 & 19,968 & 4,789 & 7,290 & 20,534 \\
\hline $90 \% 5 \mathrm{X}$ SNPs & 9,103 & 8,750 & 8,533 & 1,225 & 2,573 & 9,103 \\
\hline $75 \%$ 10X SNPs & 9,765 & 9,400 & 9,159 & 2,094 & 3,547 & 9,765 \\
\hline $90 \% 10 X$ SNPS & 3,923 & 3,691 & 3,490 & 489 & 1,224 & 3,923 \\
\hline $75 \%$ 20X SNPs & 4,069 & 3,832 & 3,624 & 703 & 1,415 & 4,069 \\
\hline $90 \%$ 20X SNPs & 1,431 & 1,313 & 1,228 & 136 & 417 & 1,431 \\
\hline Run time & 88 & 95 & 59 & 93 & 89 & 88 \\
\hline
\end{tabular}




\section{Figure 2}

SNP results averaged across the three different run parameters for $d D o c e n t$ and Stacks.

(A) Red snapper, (B) Red drum, (C) Silk snapper (see Methods or Table 1 for SNP categories description). Error bars represent one standard error.
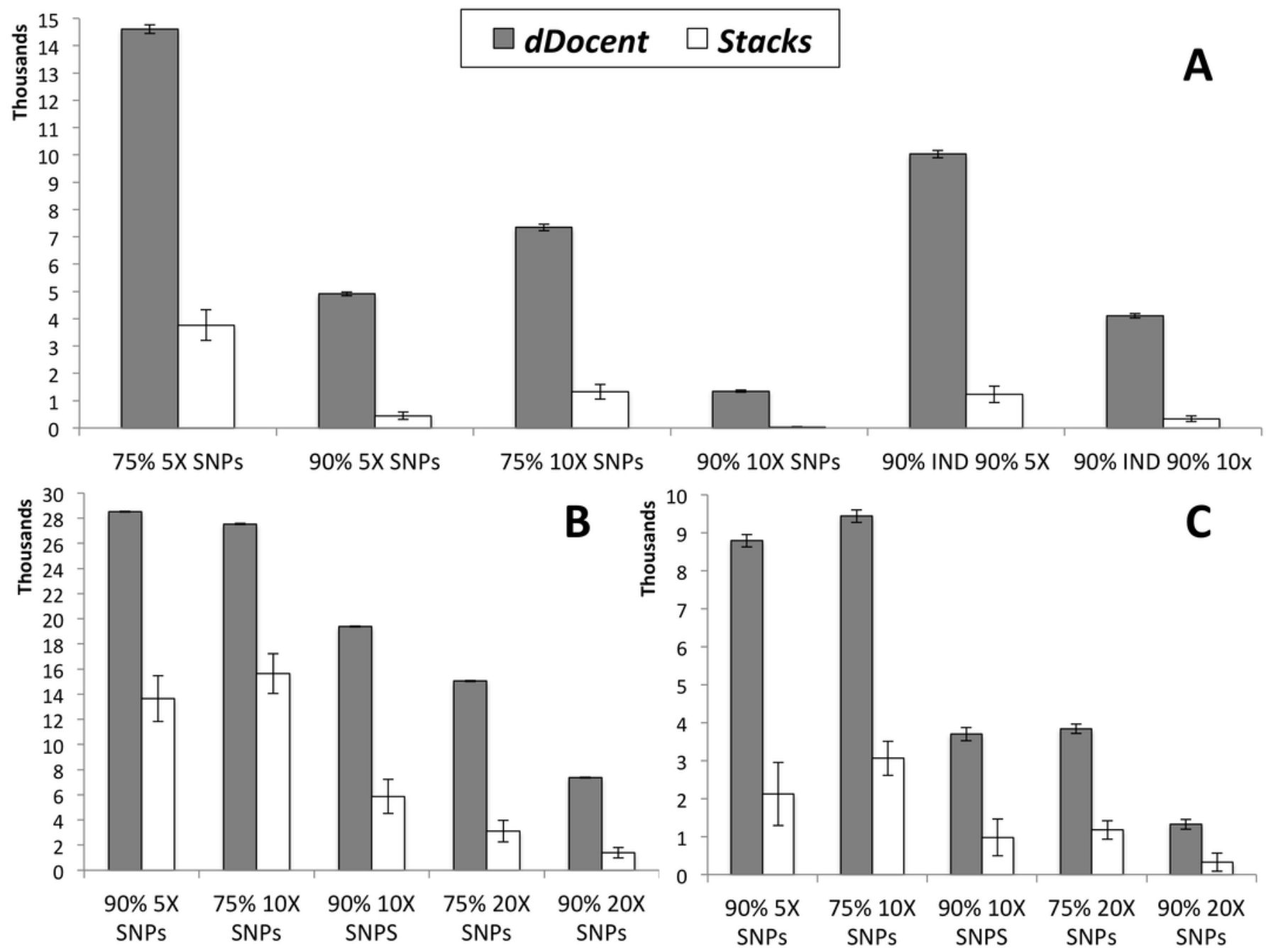